\title{
Erratum to: Dexamethasone intravitreal implant in retinal vein occlusion: real-life data from a prospective, multicenter clinical trial
}

\author{
Nicole Eter ${ }^{1}$ - Andreas Mohr ${ }^{2}$ - Joachim Wachtlin ${ }^{3}$ - Nicolas Feltgen ${ }^{4}$. \\ Andrew Shirlaw ${ }^{5}$ Richard Leaback ${ }^{5}$ For the German Ozurdex in RVO Real World \\ Study Group
}

Published online: 13 December 2016

(C) Springer-Verlag Berlin Heidelberg 2016

\section{Erratum to: Graefes Arch Clin Exp Ophthalmol DOI 10.1007/s00417-016-3431-x}

The original version of this article inadvertently contained a mistake.

Reason: authors incorrectly listed in PDF version while correctly presented in the html version (with Production). This is due to production template error.

This is being corrected in this publication.

The online version of the original article can be found at $\mathrm{http}: / / \mathrm{dx}$.doi. org/10.1007/s00417-016-3431-x.

\footnotetext{
Nicole Eter

eter@uni-muenster.de

For the German Ozurdex in RVO Real World Study Group

1 Department of Ophthalmology, University of Münster Medical School, Domagkstr 15, D-48149 Münster, Germany

2 Eye Hospital St. Joseph-Stift, Bremen, Germany

3 Augenabteilung, Sankt Gertrauden Krankenhaus, Berlin, Germany

4 University Eye Care Hospital, Göttingen, Germany

5 Allergan plc, Marlow, UK
} 